\title{
Effects of evolutionary history on adaptation in bean beetles, a model system for inquiry-based laboratories
}

\author{
Christopher W Beck ${ }^{1,2^{*}}$, Lawrence S Blumer ${ }^{3}$ and Jakob Habib ${ }^{1}$
}

\begin{abstract}
Background: Current reforms in undergraduate biology education are advancing research experiences in laboratory courses. Such experiences in evolutionary biology have largely been limited to microbial systems.

Methods: We designed a guided-inquiry experiment in which students examine the effect of evolutionary history on the potential for adaptation in the bean beetle (Callosobruchus maculatus), an insect model system widely used in evolutionary biology research. Bean beetles lay their eggs on a variety of species of dried beans (seeds of species in the Fabaceae) and the larvae develop within the bean. They are an ideal model system for studies of experimental evolution in teaching laboratories as they are easy to rear, handle and manipulate. In this study, students design an experiment to determine if adaptation to a particular bean host pre-adapts their offspring to be more successful on a new bean host.
\end{abstract}

Results: Preliminary experiments by our students suggest that beetles adapted to a lower quality host (adzuki beans, Vigna angularis) are more successful on a new higher quality host (black-eyed peas, Vigna unguiculata) than beetles adapted to a higher quality host (mung beans, Vigna radiata). However, beetles adapted to black-eyed peas are more successful on mung beans than adzuki beans.

Conclusions: Taken together, their results show that an evolutionary history on a low quality host might allow bean beetles to be more successful at invading new hosts.

Keywords: Bean beetle, Inquiry-based laboratory, Guided-inquiry, Adaptation, Evolutionary history

\section{Background}

In the recent Vision and Change report, evolution is considered one of five core concepts for biological literacy and the ability to apply the process of science through activities, such as research in laboratory courses, is considered a core competency (AAAS 2011). Yet, undergraduate biology students hold a variety of misconceptions about adaptation and natural selection (e.g., Abraham et al. 2009; Bishop and Anderson 1990; Nehm and Reilly 2007). Furthermore, many evolution laboratory activities are simulations or games that do not reflect the research practices of evolutionary biologists (Pievani and Serrelli 2008).

\footnotetext{
* Correspondence: christopher.beck@emory.edu

'Department of Biology, Emory University, 1510 Clifton Rd., Atlanta, GA 30322, USA

${ }^{2}$ School of Biological Sciences, Flinders University, GPO Box 2100, Adelaide, SA, Australia

Full list of author information is available at the end of the article
}

Many of the recent advances in the development of laboratory activities in evolutionary biology have focused on using computer simulations or virtual laboratories to address common student misconceptions related to evolution and natural selection (Abraham et al. 2009; Rands 2010; Speth et al. 2009; Toth et al. 2009). For example, in the Darwinian Snails exercise that is a part of the EvoBeaker collection, students examine the conditions for evolution by natural selection in a virtual world in which European green crabs are feeding on flat periwinkle snails (Abraham et al. 2009). In another exercise, students examine the basis of genetic variation necessary for evolution by natural selection using virtual gel electrophoresis (Toth et al. 2009). These simulations can indeed lead to significant reductions in student misconceptions about adaptation and natural selection (Abraham et al. 2009; Speth et al. 2009).

\section{슬}

(c) Beck et al.; licensee Springer. This is an Open Access article distributed under the terms of the Creative Commons Attribution License (http://creativecommons.org/licenses/by/2.0), which permits unrestricted use, distribution, and reproduction in any medium, provided the original work is properly cited. 
However, in most cases, evolution simulations do not address the need for authentic research experiences in evolutionary biology in undergraduate laboratories. An exception is Avida-ED, which is based on the Avida system that is widely used in research (Speth et al. 2009). Frasier and Roderick (2011) found that integrating research projects that were designed and implemented by their students was an important component of their reform of an undergraduate evolution course. In cases in which research experiences in evolutionary biology are integrated in a laboratory course, bacteria are typically used as the model system, given the short generation time, large population sizes and ease of manipulation (Brockhurst 2010; Frasier and Roderick 2011). To expand the range of available model systems for undergraduate evolutionary biology laboratories, we designed a guided-inquiry experiment in which students examine the effect of evolutionary history on the potential for adaptation in the bean beetle (Callosobruchus maculatus), an insect model system widely used in evolutionary biology research.

\section{Bean beetles as a model system}

The bean beetle has become well-established as a model system for inquiry-based laboratory exercises (Beck and Blumer 2007). Other inquiry-based laboratory exercises in evolutionary biology also use bean beetles (Beck and Blumer 2009; Blumer and Beck 2010). In addition to their use in teaching, bean beetles are widely used as a model system in evolutionary biology research, which allows students to connect the research that they are doing in their laboratory courses with research in the field.

Bean beetles are tropical and subtropical agricultural pest insects. Females lay their eggs on the surface of beans (seeds in the Family Fabaceae). They most commonly infest stores of mung beans (Vigna radiata), adzuki beans (Vigna angularis) or black-eyed peas (Vigna unguiculata), but can successfully emerge from other bean species (Messina et al. 2009). Eggs are deposited singly and 8 to 10 days after oviposition, a beetle larva (maggot) burrows directly from the egg into the bean. At $25^{\circ}$ to $30^{\circ} \mathrm{C}$, pupation and emergence of an adult beetle occurs 25 to 35 days after an egg was deposited. Adults are mature 24 to 36 hours after emergence and they do not need to feed. As a result, the entire life cycle can be readily and successfully completed without the provision of water or any food source other than the dried beans upon which the eggs are laid. Adults live for an average of 12 to 14 days during which time mating and oviposition occurs. Adult sexes can be distinguished by means of readily observed morphological differences that are easily seen with the naked eye. Females have dark stripes on each side of the posterior dorsal abdomen that are not found in males. Females have a lifetime egg production ranging from 30 to 100 in laboratory cultures. Because bean beetles are a stored product pest, the laboratory environment is very similar to the environment that these beetles would experience in nature. Virgin adults are easily isolated because females will lay a single egg on each bean when provided with a large number of beans on which to oviposit. Isolating single-egg beans ensures that the emerging adult is a virgin. The adults are easy to handle with forceps or a fly brush, and although they have fully functional wings, they prefer to crawl. Consequently, adults are very easy to pick from their culture container without anesthesia or special tools. Additional details on the natural history and laboratory culture of bean beetles can be found at www. beanbeetles.org/handbook.

\section{Methods}

\section{Experiments to test effects of evolutionary history}

To address student understanding of the effects of evolutionary history in adaptation, we designed two related, guided-inquiry experiments in which students examine the effect of evolutionary history on the potential for adaptation in the bean beetle (Callosobruchus maculatus) [see Additional files 1 and 2 for student handouts]. The experiments are designed as guided-inquiry activities in which students are provided with some background and the question to be addressed and then they are challenged to design experiments and generate predictions related to the hypotheses given their experimental design (D'Avanzo 1996). Students are asked to design their experiments and generate their predictions as a pre-laboratory assignment. At the beginning of class, the instructor asks the students if they have particular questions based on their prelaboratory work. Then, students work in groups to share their experimental designs and to come to a consensus design. Each group then shares their experimental design with the class and the instructor facilitates a discussion of the pros and cons of each experimental design. The class then develops a consensus experimental design that is conducted by all the students. An alternative is to allow each laboratory group to conduct their own consensus experiment, which is feasible with bean beetles as the materials are inexpensive and it is relatively easy to have a large number of beetles available for students.

Following setup of an experiment, data collection, analysis of results and interpretation of findings (described in the paragraphs below), an essential final step in any authentic research activity is the reporting and presentation of findings. Reporting and presentation activities are used for the purpose of assessing student knowledge of the scientific process and as a means of providing constructive feedback to students on the research they conducted. We have used a variety of reporting and presentation methods for this purpose, including written 
reports in scientific paper format, poster presentations coupled with one-on-one student-instructor interviews at the poster, and contributed paper format oral presentations with PowerPoint slides. Oral presentations are typically made to the entire laboratory class and provide a valuable discussion opportunity for students to question each other on their research methods, assumptions, data analyses and interpretation. Depending on the size of the class, poster and oral presentations can be done individually or in groups.

\section{Results and discussion \\ Experiment 1 \\ Experimental design}

In the first guided-inquiry experiment [see Additional file 1], students are given bean beetles that have been reared on either mung beans or adzuki beans for many generations. They are asked to design an experiment to address the question of whether adaptation to a particular host might pre-adapt a population for a switch to another host. Black-eyed peas are provided as an alternative host. Typically, students propose experiments in which females from mung bean and adzuki bean stock cultures are allowed to lay eggs on black-eyed peas. Many students also propose that they need "controls" in which females from mung bean cultures lay eggs on mung beans and females from adzuki bean cultures lay eggs on adzuki beans. Although such "controls" would allow students to compare the success of beetles on their natal hosts as compared to a novel host, they are not necessary to address the question of pre-adaptation.

Students suggest a wide range of dependent parameters to measure, including survival rate, development time, adult size and adult life span. The instructor may need to guide students to parameters that can be measured easily given the constraints of the course. For example, survival, or adult emergence success rates, can readily be tabulated as adults produce an emergence hole in the bean that is easily visible. Development time (egg to adult emergence) and body mass may be measured if students are able to check cultures daily once beetles begin emerging. However, if students are only in the laboratory once per week, development time and body mass cannot be evaluated with meaningful precision. Yet, other measures of body size that do not change with age, such as elytra length, can be measured instead. [See www.beanbeetles.org/handbook for details on linear measures of body size.] Typically, the duration of the semester precludes students measuring adult lifespan or clutch size of emerged females. On occasion, students will suggest measuring parameters that are not relevant, such as sex ratio. Students may also need to be reminded that larval development occurs within the bean so measuring larvae is not possible.
Other important considerations are the potentially confounding effects of larval competition and sexual size dimorphism on the parameters that students measure. Intraspecific competition among larvae within the same bean can result in reduced survival, extended development time, and reduced adult size at emergence (e.g., Messina 1991). As a result, beans with single eggs should be used in the experiment. Because adults emerge three to four weeks after egg-laying, the isolation of beans with single eggs can be done over the course of several laboratory periods. Females are substantially larger than males in body mass and elytra length. Therefore, it is important that students record the sex of emerging adults and analyze the data for males and females separately.

\section{Student results}

Our students designed an experiment in which females from mung bean and adzuki bean stock cultures were allowed to oviposit on black-eyed peas. Mung beans and adzuki beans are equally related to black-eyed peas (i.e., share the same most recent common ancestor) based on current phylogenies of the genus Vigna (Wang et al. 2008). However, adzuki beans are lower quality hosts than both mung beans and black-eyed peas (USDA Agricultural Research Service Nutrient Data Laboratory 2012). Students record time to adult emergence, mass at emergence and sex for beetles emerging from beans with single eggs. For both males and females, time to adult emergence was shorter if the maternal host was adzuki rather than mung (male: $\mathrm{t}=-3.46, \mathrm{df}=39, P=0.001$; female: $\mathrm{t}=-3.69, \mathrm{df}=35, P=0.001$ ) (Figure 1 ). In contrast, maternal host had only a marginally significant effect on mass at emergence for females $(\mathrm{t}=-1.93, \mathrm{df}=$ $38, P=0.061)$ and no effect in males $(\mathrm{t}=-0.36, \mathrm{df}=57$, $P=0.72$ ) (Figure 2).

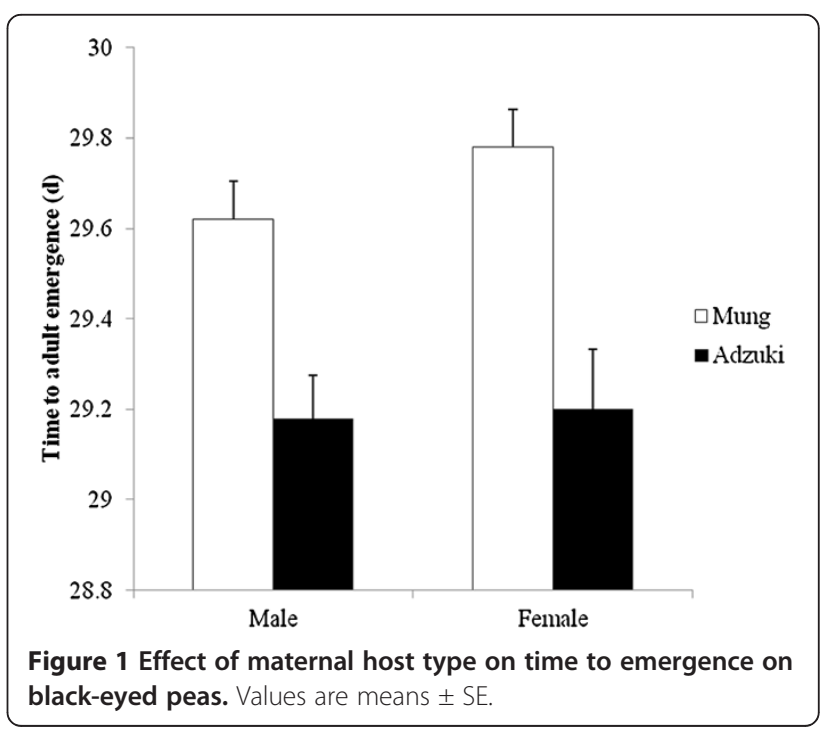




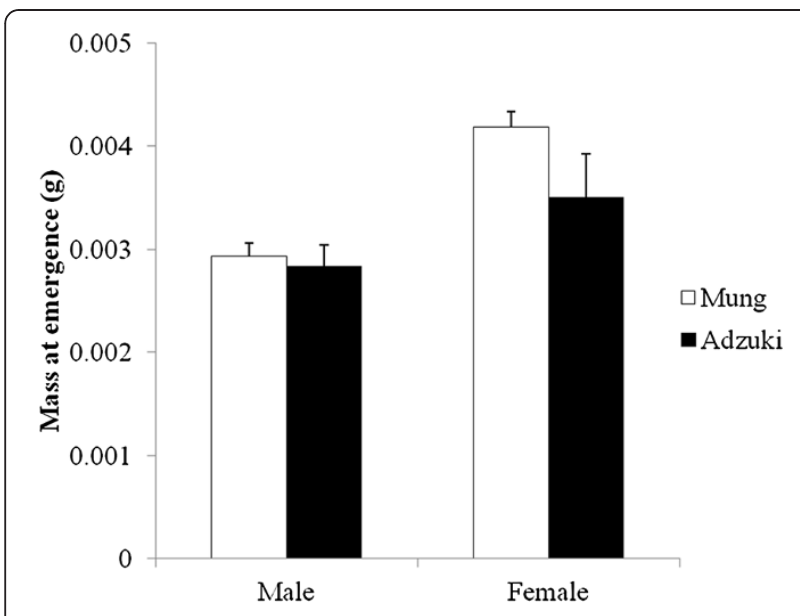

Figure 2 Effect of maternal host type on mass at emergence on black-eyed peas. Values are means $\pm \mathrm{SE}$.

In analyzing and interpreting their data from this first experiment, several students noted the possibility that the effects of previous host on offspring emerging from black-eyed peas could be due to non-genetic maternal effects. Female body size is positively correlated with egg size in bean beetles (Fox 1994b), and offspring from larger eggs develop more quickly and emerge at larger sizes than offspring from smaller eggs (Fox 1994a). Furthermore, host type is known to affect adult body size (Messina 2004); therefore, differences in the time to adult emergence could be due to differences in the size of females from the original mung and adzuki stock cultures. To control for non-genetic maternal effects, students could measure the body size of females or the size of the eggs produced and use these measures as covariates in their analysis. Another approach would be to measure traits of offspring from the second generation

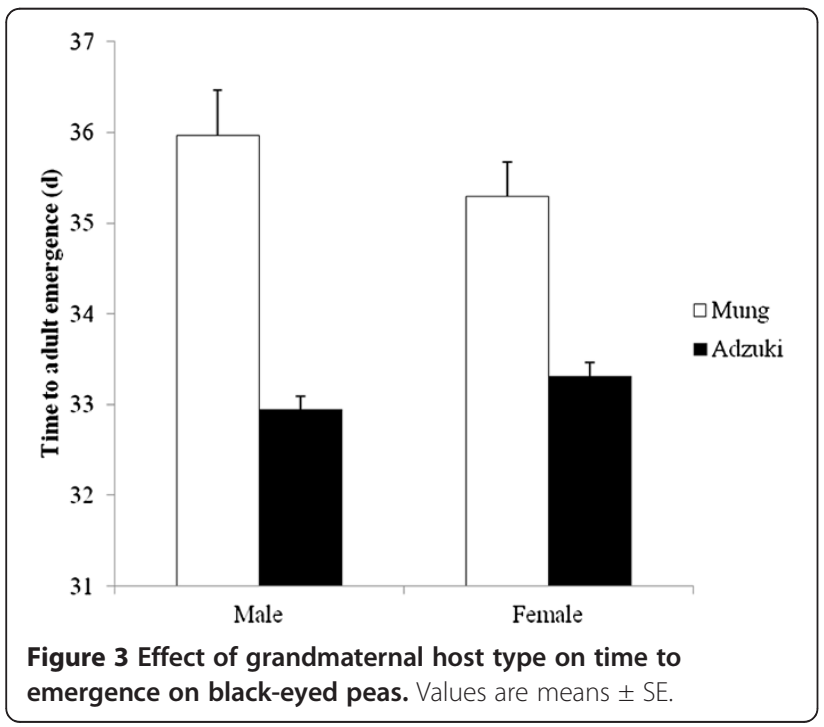

on the new host. Student data from the second generation of offspring emerging from black-eyed peas are similar to those from the first generation with original host significantly affecting time to adult emergence (male: $\mathrm{t}=5.88, \mathrm{df}=39, P<0.001$; female: $\mathrm{t}=5.04, \mathrm{df}=$ 245, $P<0.001$ ) (Figure 3 ), but not mass at emergence (male: $\mathrm{t}=-1.29, \mathrm{df}=38, P=0.21$; female: $\mathrm{t}=0.12, \mathrm{df}=$ 245, $P=0.91$ ) (Figure 4).

Both sets of results are consistent with the idea that adaptation to a low quality host (adzuki beans) pre-adapts bean beetles for a switch to a higher quality host (blackeyed peas). However, based on current genetic data, we cannot discount the possibility that adzuki beans are more genetically similar to black-eyed peas than mung beans are to black-eyed peas. In contrast to the suggestion that a low quality host pre-adapts beetles for a switch to a higher quality host, Messina and Jones (2009) found that bean beetles adapted to lentils (Lens culinaris), a low quality host, were not more likely to be successful on other hosts than beetles adapted to mung beans. Yet, in their experiment, beetles from both hosts were passaged for one generation through mung beans before being introduced to a new host. Therefore, variants in the lentil lines that were successful on mung beans were selected. No differences between lentil and mung lines were found on mung beans.

\section{Experiment 2}

\section{Experimental design}

Traditional views of plant-insect interactions suggest that insects are more likely to successfully switch to new hosts that are closely related to their original host plant, as these hosts are more likely to be similar in a range of characteristics, including the secondary compounds produced by the host plant (Agosta 2006). However, if we

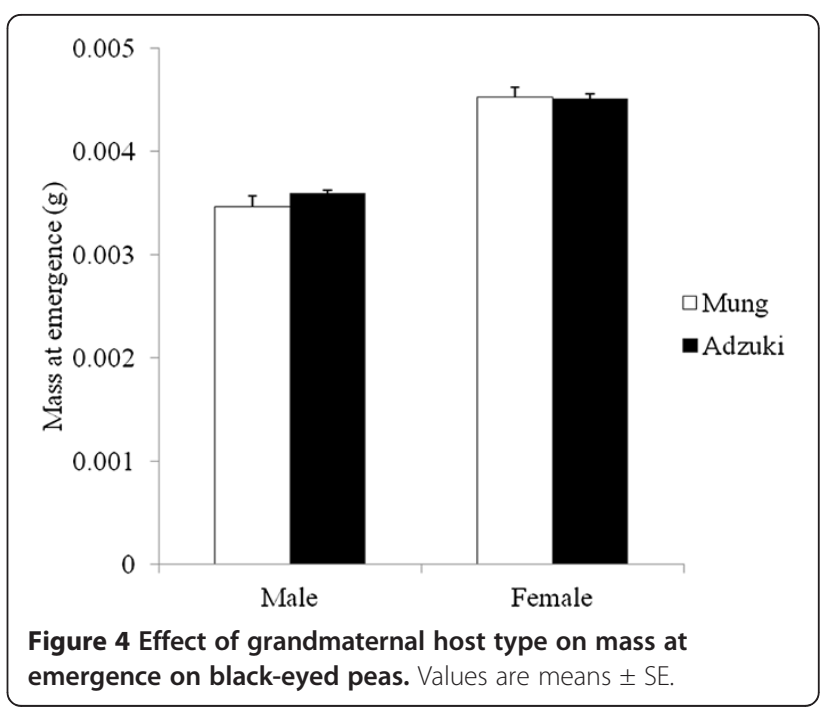




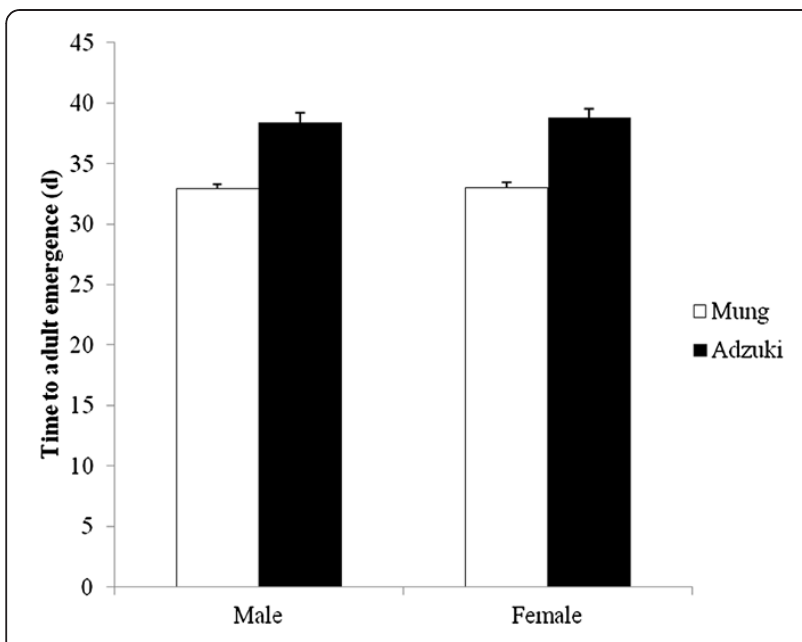

Figure 5 Effect of offspring host on time to adult emergence for beetles adapted to black-eyed peas. Values are means \pm SE.

consider variation in insect life histories in the context of resource allocation (Boggs 2009), low quality hosts might select for 'thrifty phenotypes' in terms of resource allocation. Therefore, individuals adapted to low quality hosts might more readily switch to higher quality hosts. Although the results of Experiment 1 are consistent with this thrifty phenotype hypothesis, they do not exclude the possibility of genetic similarity as the reason that beetles adapted to adzuki beans are more successful on black-eyed peas than those adapted to mung beans.

We developed a second guided-inquiry experiment [See Additional file 2] similar to Experiment 1, but provide students with the previous results from Experiment 1. In this experiment, students are asked to design an experiment to distinguish between the two hypotheses for the effects of evolutionary history on host switch success. For this experiment, students are provided with bean beetles adapted to black-eyed peas. Typically, students propose an experiment in which females from black-eyed pea stock cultures are allowed to lay eggs on either mung or adzuki beans. As with Experiment 1, many students also propose that they need controls in which females from black-eyed pea cultures lay eggs on black-eyed peas. Again, such controls would allow students to compare the success of beetles on their natal host as compared to a novel host, but they are not necessary to distinguish between the two hypotheses. Students may struggle with the predictions that would be consistent with each hypothesis, especially since the differences in the genetic similarity of mung and adzuki beans to black-eyed peas are unknown. Since beetles adapted to adzuki beans were more successful on blackeyed peas than beetles adapted to mung beans, we would expect beetles from black-eyed peas to be more successful on adzuki beans than mung beans, if genetic

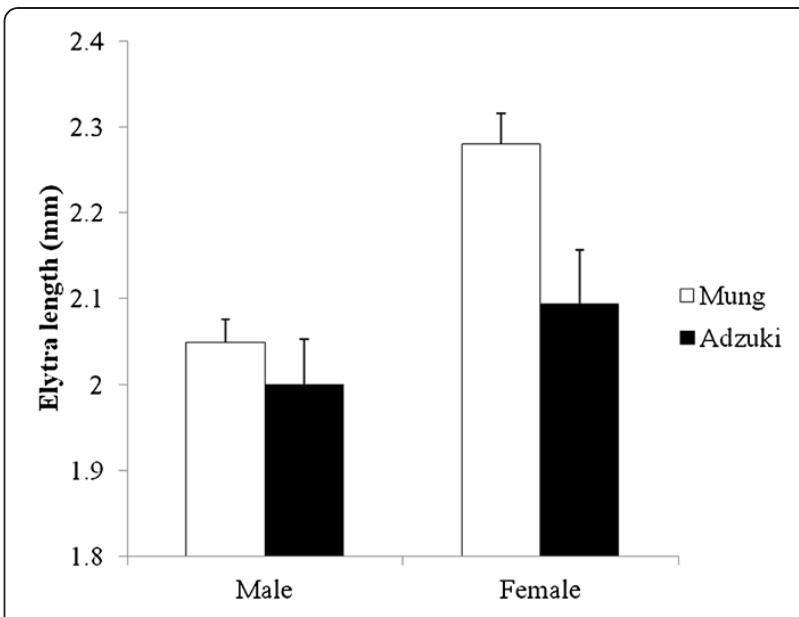

Figure 6 Effect of offspring host on adult body size for beetles adapted to black-eyed peas. Values are means \pm SE.

similarity between hosts were most important (reciprocal success in host switching between closely-related hosts). In contrast, beetles from black-eyed peas should be more successful on mung beans than adzuki beans if host quality is most important, as both mung beans and black-eyed peas are higher quality hosts than adzuki beans. The types of dependent variables often proposed by students and additional considerations that instructors should present to their students are the same as for Experiment 1.

\section{Student results}

Our students designed an experiment in which females from black-eyed pea stock cultures were allowed to oviposit on either mung or adzuki beans. They also insisted on controls in which females oviposited on black-eyed peas. Since the experiment is a guided-inquiry experiment and collecting data on the controls provides additional, albeit unnecessary data, instructors should allow students to pursue such tangents. The students collected data on emergence success, time to adult emergence, elytra length and sex.

Beetles were much more likely to successfully emerge from mung beans (68\%) than from adzuki beans (41\%) (Fisher's Exact Test, $P<0.001$ ). For both males and females, beetles developed from egg to emergence more rapidly in mung beans than in adzuki beans (male: $\mathrm{t}=-7.21 \mathrm{df}=$ 177, $P<0.001$; female: $\mathrm{t}=-7.40, \mathrm{df}=177, P<0.001$ ) (Figure 5). Females reared on mung beans were also significantly larger than those reared on adzuki beans $(t=2.61$, $\mathrm{df}=212, P=0.01$ ) (Figure 6). However, body size for males was not influenced by host type $(\mathrm{t}=0.86, \mathrm{df}=203, P=$ 0.39) (Figure 6).

Overall, the results from Experiment 1 and Experiment 2 support the thrifty phenotype hypothesis. Beetles adapted 
to a low quality host are more successful when switched to a higher quality host. However, beetles adapted to a high quality host will be more successful on other high quality hosts.

\section{Conclusions}

With a renewed call for inquiry-based learning and authentic research experiences for undergraduate students in biology (AAAS 2011) and continued misconceptions related to adaptation and natural selection (e.g., Abraham et al. 2009), the development of tractable model systems that students can use to test hypotheses related to adaptation is essential. The bean beetle is one such model system in which students may design and conduct authentic research in a laboratory course to evaluate hypotheses on the role of evolutionary history in adaptation [See Additional files 1 and 2]. Additional experiments with this model system are easily conducted by students to test hypotheses on the rates of adaptation (Beck and Blumer 2009) and the roles of natural selection and genetic drift on the rates and direction of evolutionary change (Blumer and Beck 2010). The benefits that are gained by using this model system with undergraduates may be extended readily to students in secondary and middle schools, introducing them to the process of science through guided-inquiry. The pedagogy of guided-inquiry in laboratory courses is well described [see Inquiry-based Learning link at www.beanbeetles.org] and suitable for K-12 application. Many simple and adaptable class-tested experiments with bean beetles are appropriate for secondary and middle school classrooms and are available at www.beanbeetles.org/protocols.

\section{Additional files}

Additional file 1: Student handout 1.

Additional file 2: Student handout 2.

\section{Competing interest}

The authors declare that they have no competing interests.

\section{Authors' contributions}

CB designed the study, performed the statistical analysis, and participated in writing the paper. LB participated in writing the paper. $\mathrm{JH}$ collected the data. All authors read and approved the final manuscript.

\section{Acknowledgments}

We thank the students in our courses who conducted preliminary versions of this experiment and provided feedback. This research was funded in part by National Science Foundation grants, DUE-0535903, DUE-0815135, and DUE-0814373. Any opinions, findings and conclusions or recommendations expressed in this material are those of the authors and do not necessarily reflect the views of the National Science Foundation.

\section{Author details}

${ }^{1}$ Department of Biology, Emory University, 1510 Clifton Rd., Atlanta, GA 30322, USA. ² School of Biological Sciences, Flinders University, GPO Box 2100, Adelaide, SA, Australia. ${ }^{3}$ Department of Biology, Morehouse College, 830 Westview Dr SW, Atlanta, GA 30314, USA.
Received: 8 January 2013 Accepted: 8 January 2013

Published: 26 March 2013

\section{References}

Abraham JK, Meir E, Perry J, Herron JC, Maruca S, Stal D. Addressing undergraduate student misconceptions about natural selection with an interactive simulated laboratory. Evolution: Education and Outreach. 2009;2(3):393-404.

Agosta SJ. On ecological fitting, plant-insect associations, herbivore host shifts, and host plant selection. Oikos. 2006;114(3):556-65.

American Association for the Advancement of Science (AAAS). Vision and Change: A Call to Action. AAAS, Washington DC: 2011.

Beck CW, Blumer LS. Bean beetles, Callosobruchus maculatus, a model system for inquiry-based undergraduate laboratories. In: O'Donnell MA, editor. Tested Studies for Laboratory Teaching. Proceedings of the 28th Workshop/ Conference of the Association for Biology Laboratory Education (ABLE). 2007, Association for Biology Laboratory Education. p. 274-83.

Beck CW, Blumer LS. Rapid adaptation of bean beetles to a novel host. Teaching Issues and Experiments in Ecology, 6. 2009. Experiment \#1.

Bishop BA, Anderson CW. Student conceptions of natural selection and its role in evolution. Journal of Research in Science Teaching. 1990;27(5):415-27.

Blumer LS, Beck CW. Inducing Evolution in Bean Beetles. In: Clase KL, editor. Tested Studies for Laboratory Teaching. Proceedings of the 31st Workshop/ Conference of the Association for Biology Laboratory Education (ABLE). 2010. p. $25-35$.

Boggs CL. Understanding insect life histories and senescence through a resource allocation lens. Functional Ecology. 2009;23(1):27-37.

Brockhurst MA. Using microbial microcosms to study host-parasite coevolution. Evolution: Education and Outreach. 2010;3(1):14-8.

D'Avanzo C. Three ways to teach ecology labs by inquiry: guided, open-ended, and teacher-collaborative. Bulletin of the Ecological Society of America. 1996;77(2):92-3

Fox CW. The influence of egg size on offspring performance in the seed beetle, Callosobruchus maculatus. Oikos. 1994a;71(2):321-5.

Fox CW. Maternal and genetic influences on egg size and larval performance in a seed beetle (Callosobruchus maculatus) - multigenerational transmission of a maternal effect. Heredity. 1994b;73(5):509-17.

Frasier TR, Roderick C. Improving how evolution is taught: facilitating a shift from memorization to evolutionary thinking. Evolution: Education and Outreach. 2011:4(2):298-307.

Messina FJ. Life-history variation in a seed beetle - adult egg-laying vs larval competitive ability. Oecologia. 1991;85(3):447-55.

Messina FJ. Predictable modification of body size and competitive ability following a host shift by a seed beetle. Evolution. 2004;58(12):2788-92.

Messina FJ, Jones JC. Does rapid adaptation to a poor-quality host by Callosobruchus maculatus (F.) cause cross-adaptation to other legume hosts? Journal of Stored Products Research. 2009:45(3):215-9.

Messina FJ, Mendenhall M, Jones JC. An experimentally induced host shift in a seed beetle. Entomologia Experimentalis et Applicata. 2009;132(1):39-49.

Nehm RH, Reilly L. Biology majors' knowledge and misconceptions of natural selection. Bioscience. 2007;57(3):263-72.

Pievani T, Serrelli E. Education in evolution and science through laboratory activities. Evolution: Education and Outreach. 2008;1(4):541-7.

Rands SA. Considering adaptation and the "function" of traits in the classroom, Using Wiki Tools. Evolution: Education and Outreach. 2010;3(4):633-40.

Speth EB, Long TM, Pennock RT, Ebert-May D. Using Avida-ED for teaching and learning about evolution in undergraduate introductory biology courses. Evolution: Education and Outreach. 2009;2(3):415-28.

Toth EE, Brem SK, Erdos G. "Virtual Inquiry": teaching molecular aspects of evolutionary biology through computer-based inquiry. Evolution: Education and Outreach. 2009;2(4):679-87.

USDA Agricultural Research Service Nutrient Data Laboratory. USDA National Nutrient Database for Standard Reference. 2012. http://www.nal.usda.gov/ fnic/foodcomp/search/. Accessed March 1, 2012.

Wang ML, Barkley NA, Gillaspie GA, Pederson GA. Phylogenetic relationships and genetic diversity of the USDA Vigna germplasm collection revealed by genederived markers and sequencing. Genetics Research. 2008;90(6):467-80.

doi:10.1186/1936-6434-6-5

Cite this article as: Beck et al.: Effects of evolutionary history on adaptation in bean beetles, a model system for inquiry-based laboratories. Evolution: Education and Outreach 2013 6:5. 